\title{
Electro-Optic Quantum Memory for Light Using Two-Level Atoms
}

\author{
G. Hétet, ${ }^{1}$ J. J. Longdell, ${ }^{2,3}$ A. L. Alexander, ${ }^{2}$ P. K. Lam, ${ }^{1}$ and M. J. Sellars ${ }^{2, *}$ \\ ${ }^{1}$ ARC COE for Quantum-Atom Optics, Australian National University, Canberra, ACT 0200, Australia \\ ${ }^{2}$ Laser Physics Centre, RSPhysSE, Australian National University, Canberra, ACT 0200, Australia \\ ${ }^{3}$ Department of Physics, University of Otago, Dunedin, New Zealand
}

(Received 12 March 2007; published 16 January 2008)

\begin{abstract}
We present a simple quantum memory scheme that allows for the storage of a light field in an ensemble of two-level atoms. The technique is analogous to the NMR gradient echo for which the imprinting and recalling of the input field are performed by controlling a linearly varying broadening. Our protocol is perfectly efficient in the limit of high optical depths and the output pulse is emitted in the forward direction. We provide a numerical analysis of the protocol together with an experiment performed in a solid state system. In close agreement with our model, the experiment shows a total efficiency of up to $15 \%$, and a recall efficiency of $26 \%$. We suggest simple realizable improvements for the experiment to surpass the no-cloning limit.
\end{abstract}

DOI: 10.1103/PhysRevLett.100.023601

PACS numbers: $42.50 . \mathrm{Gy}, 42.50 . \mathrm{Md}$

Some of the most significant advances in quantum information processing have been made using quantum optics. To extend these techniques, it is necessary to have devices such as single photon sources and quantum repeaters using quantum memories, where information is exchanged in a controlled fashion between light fields and material systems. A quantum memory for light is a device that can efficiently delay or store the quantum states of light fields. This is usually achieved via some form of imprinting onto an atomic system. The quantum states stored must also be faithfully retrievable on demand and the total efficiency of the processes must exceed the classical benchmark so that quantum information can be retained [1]. It has been proposed that the requisite control and strong coupling can both be achieved using an ensemble approach, where the light field interacts with a large number of identical atoms [2]. Classical pulses [3] and single photon states [4] have been stored and retrieved using electromagnetically induced transparency from warm vapor cells and magneto-optical traps. In the continuous variable regime, quantum states of light have been mapped onto atoms using the off resonant interaction of light with spin polarized cesium vapors [5]. Further improvements are nevertheless still needed for realizing an efficient and reliable quantum memory.

In 2001, Moiseev and Kröll [6] published a proposal for a quantum memory for light based on modified photon echoes. In contrast to a normal photon echo [7-9], the rephasing came from controlled reversible inhomogeneous broadening (CRIB). This proposal has then been generalized to other broadening mechanisms [10-12]. Storage of multiple pulses using CRIB has been demonstrated using Stark shifts in europium dopants $[13,14]$ and work towards demonstrating such echoes in other systems has been carried out $[11,15]$. The quantum memory proposals using CRIB operate via a time reversal of the storage process. It has been shown that by reversing the detunings of the atoms, the equations of motion for light traveling in the backward direction describe the motion of a time reversed copy traveling in the forward direction [10]. The pulse can exit the ensemble in the backward direction by applying a phase matching operation to the atoms with an auxiliary third atomic level and $\pi$ pulses. Sangouard et al. [16] have recently shown that a quantum memory can be achieved without any external fields. However, using this approach, the efficiency would be limited to a maximum of 54\%.

In this Letter, we show that $100 \%$ efficiency using CRIB is possible using only two level atoms Stark shifted by an external electric field. The only light seen by the atomic ensemble during the entire process is then the light field of interest and the echo propagates in the forward direction without being reabsorbed provided the Stark shift is linear along the sample length. The principle benefit of such a two-level scheme lies in its simplicity. First, the absence of phase matching $\pi$ pulses greatly simplifies the implementation. The precision of the electro-optic switching is not as critical as the $\pi$ pulse parameters. The scheme is also more robust than those using optic-optic interactions, where cross-coupling and transverse-modal effects on the beams may reduce the process efficiency. Second, as the memory requires only two atomic levels, this scheme is applicable to many more atomic systems. In particular, erbium dopants which allow operation at the telecommunication wavelength of $1.5 \mu \mathrm{m}$ have been shown to have very good two-level characteristics [17], while a lambda system has yet to be demonstrated.

To demonstrate the efficiency of the memory, we consider the interaction between a collection of $\mathrm{N}$ two-level atoms and a quantum optical field with slowly varying envelope $\hat{\mathcal{E}}(z, t)$. As shown in Fig. 1(a), the pulse with duration $t_{\text {pulse }}$ enters the medium at $z=-z_{0}, t=-t_{0}$ and the detuning of the atoms are flipped at $t=0$. We follow the same procedure as in Ref. [18] and use locally averaged atomic operators. The Heisenberg-Langevin 
(a)
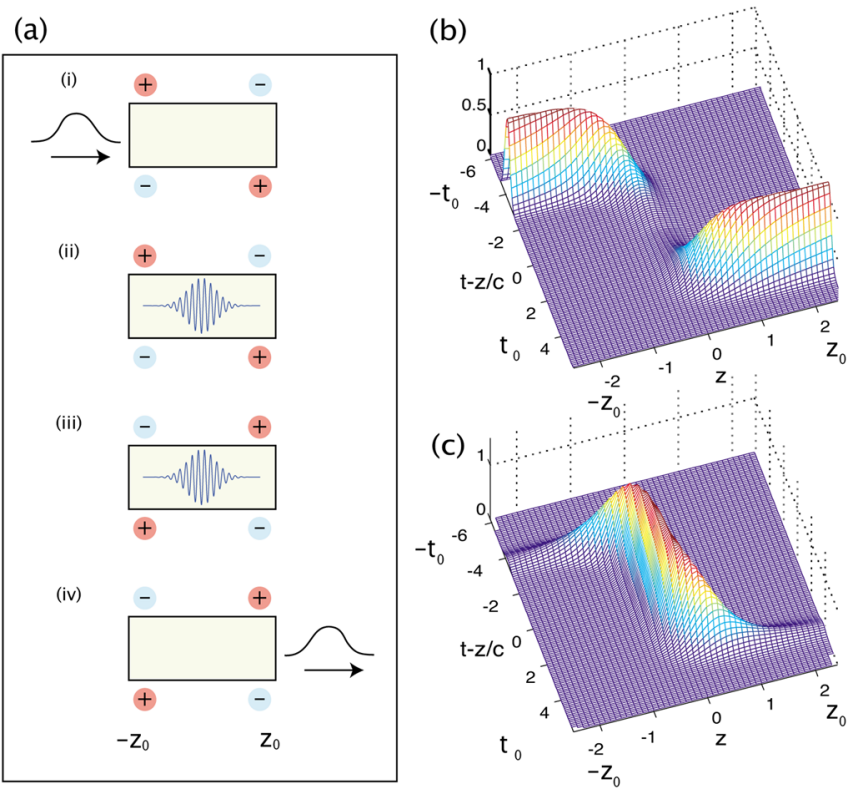

FIG. 1 (color online). (a) Schematic of the electro-optic quantum memory. (i) The input light field enters the sample at $z=$ $-z_{0}$ and $t=-t_{0}$; (ii) the light field is imprinted onto the broadened two-level atomic ensemble, (iii) to recall the light field, the polarity of the quadrupole electric field is flipped at $t=0$, (iv) an output field is retrieved at $z=z_{0}$ and $t=t_{0}$. With no decoherence $\gamma=0$, and the optical depth chosen to be $g N / \eta=10 / 3$, (b) and (c) show the space-time grid plots of the light field intensity and the atomic polarization, respectively. The input pulse duration is $t_{\text {pulse }}=t_{0} / 4$ and the quadrupole induced broadening is $2 / t_{\text {pulse }}$.

equations describing the interaction in a moving frame at the speed of light are

$$
\begin{gathered}
\dot{\hat{\alpha}}=-(\gamma / 2 \pm i \eta z) \hat{\alpha}+i g \hat{\mathcal{E}}\left(\hat{\sigma}_{g}-\hat{\sigma}_{e}\right)+\hat{F}_{\alpha} \\
\dot{\hat{\sigma}}_{g}=\gamma \hat{\sigma}_{e}+i g\left(\hat{\mathcal{E}}^{\dagger} \hat{\alpha}-\hat{\mathcal{E}} \hat{\alpha}^{\dagger}\right)+\hat{F}_{g} \\
\partial_{z} \hat{\mathcal{E}}=i g N \hat{\alpha}
\end{gathered}
$$

where $\hat{\alpha}$ is the atomic polarization operator, $\hat{\sigma}_{e / g}$ the population of the excited or ground state, $g$ the atomic transition coupling strength, and $i \eta z$ is the linearly varying detuning from resonance. We also introduced $\gamma$ as a decay rate from the excited state and the corresponding Langevin operators. Under a small pulse approximation, ensuring that a negligible amount of atoms reaches the excited state $\left(\sigma_{e} \ll \sigma_{g}\right)$, the nonzero noise correlations are found to be [19]

$$
\left\langle\tilde{F}_{\alpha}\left(z_{1}, t_{1}\right) \tilde{F}_{\alpha}^{\dagger}\left(z_{2}, t_{2}\right)\right\rangle=2 \gamma \frac{\delta\left(z_{1}-z_{2}\right) \delta\left(t_{1}-t_{2}\right)}{n \mathcal{A}},
$$

where $\mathcal{A}$ is the cross section area of the beam and $n$ the atomic density. Because the spontaneous excitation of atoms to the excited states requires large energy, the noise arises from the normally ordered Langevin correlations and is therefore vacuum noise [19]. Furthermore, the Heisenberg-Langevin equations are linear after the weak probe approximation so the atomic and optical field variables can be treated as $c$ numbers [18]. These two results ensure that transmissivity is the only quantity needed to fully characterize the memory.

The effective optical depth of the sample is $g N / \eta$. This ratio indeed quantifies the portion of the input light that is not stored in the sample, a feature of the absorption of short pulses by narrow linewidth atoms [20]. $2 \eta z_{0}$ on the other hand gives the spectral coverage of the absorption process. We will here let $g N / \eta=10 / 3$ and $2 \eta z_{0}=2 / t_{\text {pulse }}$ to ensure an optimum writing efficiency. The sign of the Stark shift will be reversed after the complete absorption of the pulse. Figures 1(b) and 1(c) show the results of numerical simulations with a zero decay rate $\gamma=0$. Figure 1(b) reveals that with these parameters, a large echo comes out of the sample after flipping the electric field. The excitation in the medium can be thought of as a polariton asymptotically slowing down to zero velocity with the asymptotic limit for the different spectral components of the pulse distributed spatially along the propagation direction. When the sign of the Stark shift is reversed, the polariton accelerates in the forward direction out of the sample. Because of the monotonicity of the Stark shift with space, all the spectral components of the pulse escape the sample without being resonant with the atoms. A detailed description of this light-matter superposition in this scheme will be presented elsewhere.

The importance of the Stark-shift monotonicity is highlighted further in Figs. 2(a) and 2(b). The figures show plots of the electro-optic memory efficiencies as a function of optical depth. It can be seen in Fig. 2(a) that the efficiency of our memory asymptotes to $100 \%$ with optical depth. It was shown in Ref. [16] however, that the efficiency of such a two-level atom electro-optic memory is limited when the broadening is nonmonotonic. To allow a direct comparison between the two-level atom technique analyzed in Ref. [16] and our scheme, we show in Fig. 2(b)
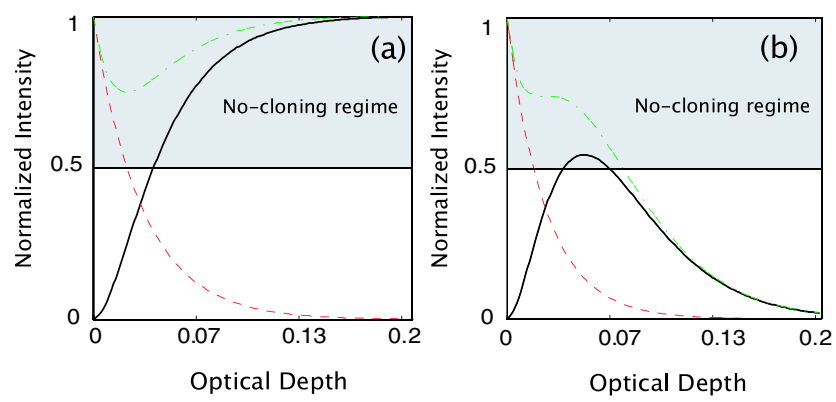

FIG. 2 (color online). Memory efficiency as a function of optical depth when (a) the Stark shift is linear with position and (b) when the Stark shift is nonmonotonic. Solid lines represent the efficiency of the memory; dashed lines, the fraction of transmitted light; and dot-dashed lines, the total energy exiting the medium. Shaded regions are the no-cloning regimes. 
the result of simulations using a nonmonotonic broadening. A complete agreement with the results of Ref. [16] is found. The efficiency reaches a maximum of $54 \%$ and at high optical depths, the optical information is retained in the medium. Our modeling also demonstrates that a small spatial nonlinearity of the Stark shift, $\eta z$, and finite switching speed of the applied electric field have very little influence on the efficiency. As the efficiency of our electro-optic memory can be well above $50 \%$ with no excess noise, the echo is guaranteed to be the best possible copy of the input state [21], demonstrating that our scheme is a quantum memory for light.

Figure 3 shows a contour map of the real part of the electric field. Because of the large phase shift seen by the field when it enters the medium, the last atoms in the sample absorb the field a long time after the first atoms. The storage time is then required to be large enough for the atoms to reradiate the whole input pulse. Figure 3(a) presents simulations where the storage time is 4 times the pulse duration. In that situation, a time varying phase shift is present across the output pulse, so that it is frequency shifted with respect to the input. Figure 3(b) shows simulations where the Stark shift is flipped after nearly all the atoms have absorbed the field. The frequency shift is then eliminated and only a constant phase shift is present. One way of compensating for the phase shift would be to cascade two electro-optic memories using opposite switching procedures. Another method would be to use an electro-optic phase shifter driven with the appropriate voltage waveform.

The initial demonstrations of photon echoes via CRIB used a linear Stark shift [13], as in the current proposal. At the time it was thought that a more difficult experiment involving an auxiliary atomic level and counter propagat-

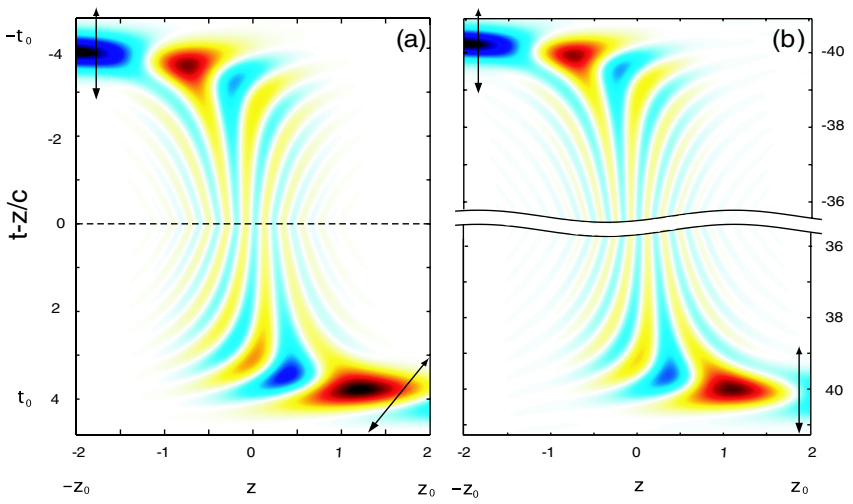

FIG. 3 (color online). Real part of the optical field in a moving frame at $c$. At $t=-t_{0}$, the light field enters the sample and is gradually absorbed by the medium. At $t=0$, the quadrupole field is flipped and the time reverse process commences producing a forward propagating pulse. For the parameters given in Fig. 1, with a storage time of $8 t_{\text {pulse }}$, (a) shows a small phase shift across the retrieved pulse. With a storage time $80 t_{\text {pulse }}$, (b) shows a near ideal pulse retrieval. The arrows denote the wave front of the light fields. ing $\pi$ pulses would be required to achieve a quantum memory for light. The above analysis shows that this is not the case. Here we report enhancements of the efficiency by more than 5 orders of magnitudes compared with the initial Stark-echo demonstrations of Ref. [13] in complete agreement with the theory. A large part of this improvement is due to a change of the dopant ions used to praseodymium allowing larger optical depths to be reached.

The experiment was carried out on a spectral antihole which was prepared as described in Fig. 4. Light from a highly stabilized dye laser was frequency shifted and gated with acousto-optic modulators. The pulse was then steered toward the sample of $\operatorname{Pr}^{3+}: \mathrm{Y}_{2} \mathrm{SiO}_{5}(0.05 \%)$. The sample was approximately a $4 \mathrm{~mm}$ cube and was held at temperatures in the range $2-4 \mathrm{~K}$. Four electrodes were placed around the sample in a quadrupole arrangement and provided an electric field that varied linearly along the optical path. The electrodes were $1.7 \mathrm{~mm}$ diameter rods separated by $8 \mathrm{~mm}$. Voltages of approximately $\pm 5 \mathrm{~V}$ were used to broaden the antihole and were able to be switched in $1 \mu \mathrm{s}$. Heterodyne detection was used to detect the transmitted pulses. The beam diameter in the sample was approximately $200 \mu \mathrm{m}$ and the corresponding pulse areas $\pi / 20$.

Figure 5 shows the experimental traces of the electrooptic echo memory with and without the preparation of the two-level antihole. In the results, $49 \%$ of the incident light was transmitted straight through the sample (with less than $2 \%$ absorption without the antihole) and $15 \%$ of the total input light was recalled as an echo. The recall efficiency, defined as the ratio between the reemitted and absorbed

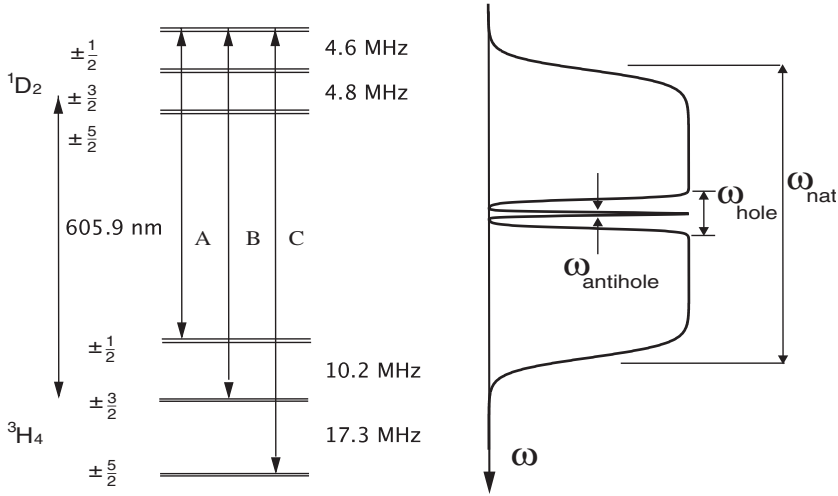

FIG. 4. Energy level diagram and spectral scheme for the praseodymium dopants in yttrium orthosilicate $\mathrm{Pr}^{3+}: \mathrm{Y}_{2} \mathrm{SiO}_{5}(0.05 \%)$. The light at frequency $A$ is the light stored by the electro-optic memory. The natural inhomogeneous linewidth of the sample, $\omega_{\text {nat }}$, is a few $\mathrm{GHz}$ wide. To set up the experiment, the applied light is swept around frequency $A$ to create a spectral hole a few $\mathrm{MHz}$ wide, $\omega_{\text {hole }}$. Light at frequencies $B$ and $C$ is then applied to prepare a narrow antihole around $A$ with linewidth $\omega_{\text {anti }}$. Although the diagram shows the use of the $\pm 1 / 2$ excited states, there are ions contributing to the antihole from the $\pm 3 / 2$ and $\pm 5 / 2$ excited states due to inhomogeneous broadening in the optical transition. In our experiment $\omega_{\text {antihole }}=30 \mathrm{kHz}$. 


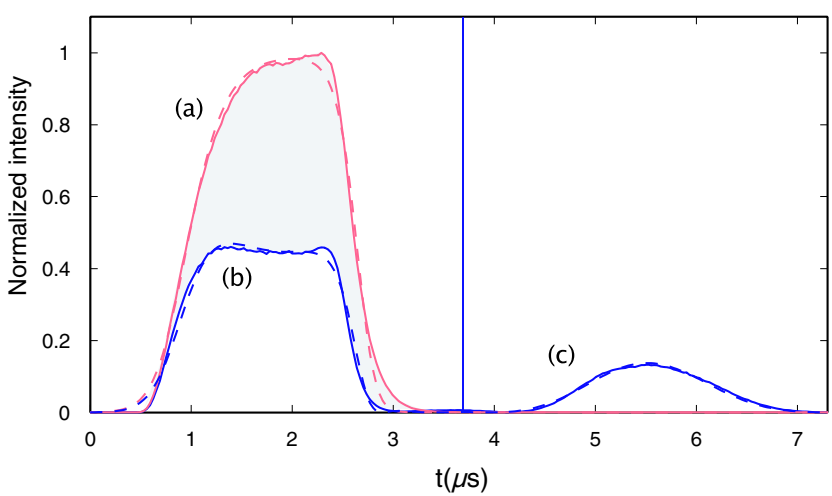

FIG. 5 (color online). Experimental results (solid lines) and numerical simulations (dashed lines) of the electro-optic echo setup. Trace (a) shows the input pulse detected after transmission through the sample when no antihole is prepared. Trace (b) shows the transmitted pulse and (c) the stored-and-recalled pulse components. The vertical line at $3.7 \mu$ s denotes the time at which the polarity of the Stark-shifting electric field is flipped.

light is $26 \%$, showing the potential improvements of our system with larger optical depths. A minimal output pulse distortion was observed, which demonstrates the rather large time-bandwidth product in our experiment. Using our numerical model, we vary the spectral width of the unbroadened antihole and the optical depth of the sample to match the experimental results. Close agreement between the experimental results and the simulations is obtained only with these two free parameters. Our numerical model suggests an antihole width of $30 \mathrm{kHz}$, in agreement with the experimental expectation if the hyperfine transition broadening were the main limitation to the antihole width.

For a given pulse length, the optimization of the experiment is dependent on a compromise between increasing the ratio between applied and intrinsic broadenings and maximizing the optical depth of the sample. In our experiment, $2 \eta z_{0} / \gamma$ is around 12 while the optical depth $g N / \eta=0.06$. For each crystallographic site where praseodymium is located, there is another related to it by inversion. In order to implement a completely efficient memory, only one of the site pair can be used. In principle, this could be achieved by Stark shifting with a homogeneous electric field and optical pumping. In our experiment, however, both orientations were used. The theoretical modeling on Fig. 5 takes into account these two orientations by having two Bloch equations and two source terms for the optical field. Simulations suggest that using Fourier limited pulses, selecting only one orientation of the praseodymium ions, and increasing the optical depth by a factor of 3 would enable the scheme to reach more than $50 \%$ efficiency.

In conclusion, we have proposed an electro-optic quantum memory for light with a linear Stark shift. In contrast to existing quantum memories based on controlled inhomogeneous broadening, our scheme requires only two atomic levels and is therefore applicable to a wide range of systems. Moreover, our scheme does not require auxiliary optical pulses for the imprint and recall process. Our experiments show an efficiency of $15 \%$ and a timebandwidth product of around three which compares favorably with the performance of quantum memories based on EIT [4]. The experiment is well modeled by the MaxwellBloch equations. Modest improvements on the experimental parameters will allow efficiencies higher than $50 \%$ to be achieved.

We thank C. Simon and B.C. Buchler for useful discussion. We acknowledge financial assistance from the Australian Research Council.

*matthew.sellars@anu.edu.au

[1] K. Hammerer, M. M. Wolf, E. S. Polzik, and J. I. Cirac, Phys. Rev. Lett. 94, 150503 (2005).

[2] A. E. Kozhekin, K. Molmer, and E. Polzik, Phys. Rev. A, 62, 033809 (2000), and references therein.

[3] C. Liu, Z. Dutton, C. H. Behroozi, and L. V. Hau, Nature (London) 409, 490 (2001); D. F. Phillips, A. Fleischhauer, A. Mair, R. L. Walsworth, and M. D. Lukin, Phys. Rev. Lett. 86, 783 ( 2001).

[4] T. Chanelière et al., Nature (London) 438, 833 (2005); M. D. Eisaman et al., Nature (London) 438, 837 (2005).

[5] B. Julsgaard, J. Sherson, J. I. Cirac, J. Fiurasek, and E. S. Polzik, Nature (London) 432, 482 (2004).

[6] S. A. Moiseev and S. Kroll, Phys. Rev. Lett. 87, 173601 (2001).

[7] N. A. Kurnit, I. D. Abella, and S. R. Hartmann, Phys. Rev. Lett. 13, 567 (1964).

[8] T. W. Mossberg, Opt. Lett. 7, 77 (1982).

[9] N. W. Carlson, L. J. Rothberg, A. G. Yodh, W. R. Babbitt, and T. W. Mossberg, Opt. Lett. 8, 483, (1983).

[10] B. Kraus et al., Phys. Rev. A 73, 020302(R) (2006).

[11] M. Nilsson and S. Kroll, Opt. Commun. 247, 393 (2005).

[12] S. A. Moiseev, V. F. Tarasov, and B. S. Ham, J. Opt. B 5, S497 (2003).

[13] A. L. Alexander, J. J. Longdell, M. J. Sellars, and N. B. Manson, Phys. Rev. Lett. 96, 043602 (2006).

[14] A. L. Alexander, J. J. Longdell, M. J. Sellars, and N. B. Manson, J. Lumin. 127, 94 (2007).

[15] M. U. Staudt et al., Opt. Commun. 266, 720 (2006).

[16] N. Sangouard et al., Phys. Rev. A 75, 032327 (2007).

[17] T. Böttger, C. W. Thiel, Y. Sun, and R. L. Cone, Phys. Rev. B 73, 075101 (2006).

[18] M. Fleischhauer and M.D. Lukin, Phys. Rev. Lett. 84, 5094 (2000).

[19] C. Cohen-Tannoudji, J. Dupon-Roc, and G. Grynberg, Atom-Photon Interactions (Wiley, New York, 1992).

[20] M. D. Crisp, Phys. Rev. A 1, 1604 (1970).

[21] F. Grosshans and P. Grangier, Phys. Rev. A 64, 010301(R) (2001). 